\title{
Structural Mechanics Analysis Using an FE-Mesh Adaption to Real, 3D Surface Detected Geometry Data
}

\author{
Sebastian Katona ${ }^{1}$, Michael Koch ${ }^{1}$, Tobias C. Spruegel ${ }^{2}$ and Sandro Wartzack ${ }^{2}$ \\ 1. Department of Mechanical Engineering and Building Services Engineering, Technische Hochschule Nuernberg Georg Simon Ohm, \\ Nuremberg 90489, Germany \\ 2. Chair of Engineering Design KTmfk, Friedrich-Alexander-Universitaet Erlangen-Nuernberg, Erlangen 91058, Germany
}

\begin{abstract}
Within today's product development process, various FE-simulations (finite element) for the functional validation of the desired characteristics are made to avoid expensive testing with real components. Those simulations are performed with great effort for discretization, use of simulations conditions, like taking different non-linearities (i.e., material behavior, etc.) into account, to create meaningful results. Despite knowing the effects of deformations occurring during the production processes, always the non-deformed design model of a CAD-system (computer aided design) is used for the FE-simulations. It seems rather doubtful that further refinement of simulation methods makes sense, if the real manufactured geometry of the component is not considered for in the simulation. For an efficient exploit of the potential of simulation methods, an approach has been developed which offers a geometry model for simulation based on the existing CAD-model but with integrated production deviations as soon as a first prototype is at hand by adapting the FE-mesh to the real, 3D surface detected geometry.
\end{abstract}

Key words: FEA (finite element analysis), preprocessing, simulation, 3D surface detection, RE (reverse engineering).

\section{Introduction}

Today's product development process is picking up the pace. To avoid performing complex and expensive testing methods including all iterations of the product development with a real prototype, various FE-simulations (finite element) for functional validation of desired characteristics are made. The discretization of a developed component and the use of the simulation conditions, like taking different non-linearities (i.e., material behavior, contact situations, large deflections, etc.) into account, are a tremendous effort and mandatory to get precise and significant results.

In general, every produced component shows differences to its ideal geometry of the designed CAD-model (computer aided design), i.e., discrepancies due to deformations in the production process (Fig. 1).

Corresponding author: Sebastian Katona, M. Eng., research fields: generating corrected, more realistic geometry data for simulation purposes using 3D surface detection methods. E-mail: sebastian.katona@th-nuernberg.de.
These differences can vary, depending on the manufacturing process, random deviations and the component size. In the cutting process, the value is relatively small, but using other manufacturing techniques, process-related effects may occur and can trigger bigger deformations. So, spring back [1] and drapery [2] may arise when using forming techniques (i.e., deep-drawing or bending). Besides that, using various casting processes (i.e., die casting or injection moulding), effects like shrinkage [3] and warpage, i.e., due to the solidification and cooling of the component in the die, due to separating sprue and overflow system or due to inhomogeneous temperature fields when cooling to room temperature at components with differences in wall thickness [4], can also cause large deformations. Despite knowing the effects of deformations, due to the production process mentioned above, the product developer always uses the non-deformed design model of a CAD-system for the FE-simulation of a component [5]. It seems rather doubtful that further refinement of simulation methods 


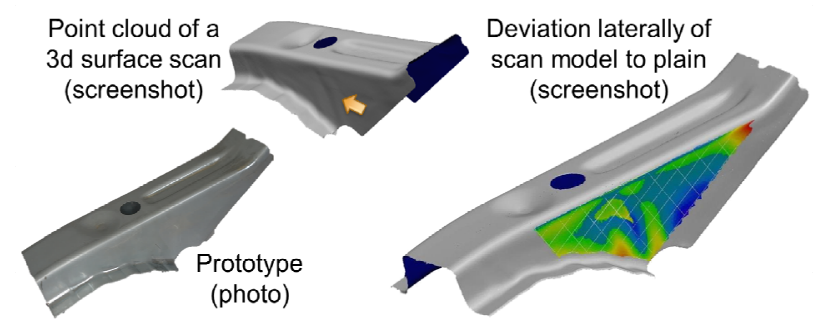

Fig. 1 Exemplarily illustration showing the deviations of a component.

will make sense if the real manufactured geometry of the component is not considered in the simulation.

One way to represent those deviations of the real geometry is the process of RE (reverse engineering). $\mathrm{RE}$ is mainly used for reconstructing geometric surfaces, starting from physically present components, in order to describe the existing form sufficiently precise [6]. Within this generic process [7], a 3D (three dimensional) surface scan of a component/prototype is created with a laser line or structured light scanner generating a point cloud, which is tessellated to a polygonal model afterwards. For getting surface or volume geometry data, a complex and time consuming reconstruction [8] to mathematically described surfaces, mostly NURBS-patches (non-uniform rational b-splines) based upon the polygonal model, must be performed [9]. NURBS-patches are of relatively small memory usage, since only control points, grid and knot vectors have to be saved. In addition, the NURBS-algorithms work fast and are numerical stable [10]. The main disadvantages to create a real geometry model within the RE-process are: the time of surface reconstruction, approximately $80 \%$ or even more [8] of the whole time in reverse engineering, is often used for this step, and, furthermore, the fact that with this CAD-model, a complete new FE-simulation containing all tasks of preprocessing like meshing or the definition of boundary conditions, must be performed once again.

Accordingly, the aim of this article is to offer a geometry model for simulation purposes based on the existing CAD-model but with integrated production deviations as soon as a first prototype is at hand to exploit the potential of a simulation method efficiently.
The following method describes an opportunity to automatically adapt existing FEA-meshes (finite element analysis), generated with the non-deformed CAD-model, to the deviated geometry caused by production. 3D surface scans and relevant algorithms are used for that purpose. The surface nodes are extracted from an ANSYS input file. A measurement of the deviation to the scanned model at each node position (either point cloud or polygonal model) takes place by using a comparison point within the software PolyWorks. Afterwards a preload-step is defined, in which the deviation of every surface node is applied as a displacement. The mesh emerging from this preload-step with its new nodes coordinates is the basis for further simulations with real geometry. Herewith, a completely new input file with the new mesh and the identical content of the initial input file based on the ideal CAD-model (considering material definition, forces, boundary conditions, etc.) is created. As a result, an FE-model with real geometry is available and can be used for simulations without spending a lot of time on additional preprocessing. The advantage can be seen in the omission of a complex design of a new model for simulating the real manufactured geometry. Comparative analysis between ideal and real geometry is simple, when using this approach in the product development process.

\section{Methodology to Identify and Evaluate the Deviations and the Use of the Approach to Adapt FE-Meshes}

To use the algorithm of the FE-mesh adoption, the deviations of the real manufactured component and the ideal CAD-model must be identified and evaluated whether they are critical or not (Fig. 2).

First of all, the standard procedure of an FE-simulation, the preprocessing, is performed. Therefore, the ideal, non-deformed CAD-model of the designed component is meshed with the proper elements within the FE-software (in this case: ANSYS). The simulation engineer needs a profound knowledge (i.e., element type, 


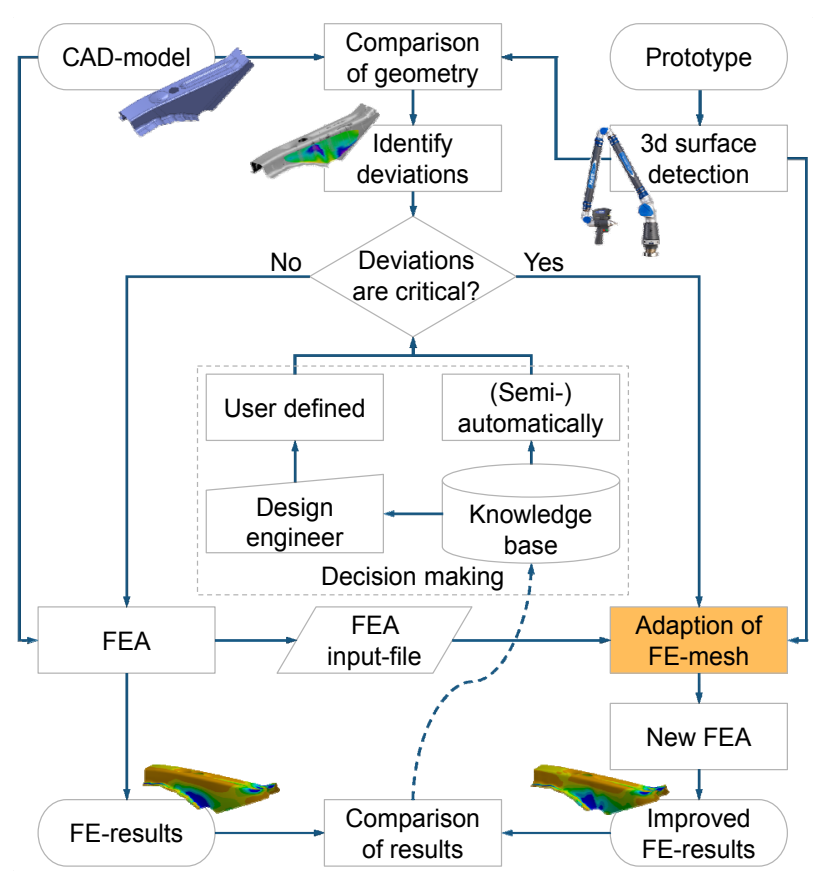

Fig. 2 Process of identification and evaluation of the deviations.

element size, etc.) and a lot of time for the creation of this mesh during the product development phase, because the mesh has a major influence on the calculated results of the analysis. In order to conduct an exact and meaningful simulation, a sufficiently fine and uniform mesh of almost undistorted, compliant elements has to be created [11]. The mesh information (number and coordinates of nodes, elements, etc.) and all further information about forces, displacements and boundary conditions specified during preprocessing are read and saved as an input-file for ANSYS. Then, a numerical solving can be performed based on this data.

Besides the standard FEA, the surface of a prototype must be detected three-dimensional to get the real manufactured shape of the component. There are mainly two ways to record a component: with a laser line scanner or a structured light scanner. In this specific case, a FARO Laser Line Probe, mounted on a FARO Platinum Arm for tracking, is used. The arm provides seven axis of rotation. Located in each joint, temperature sensors allow the arm to measure and react to thermal variations [12]. That scanner system detects the surface in $3 \mathrm{D}$ with the laser light-section method (Fig. 3). This method is based on the basic principle of triangulation [13] and offers a rasterized point cloud, which can be displayed, postprocessed and, if necessary, modified and edited with appropriate software tools, e.g., the software PolyWorks (by InnovMetric Software Inc., Quebec, QC, Canada).

As a result, the detected data and an input-file of the initial FEA enable the engineer to perform the process of FE-mesh adaption to get a new file for an FE-analysis. Then the opportunity arises to compare both simulations of the ideal and the deformed geometry. The results could be repatriated to the knowledge base to improve the decision making process for future simulation orders.

The process of adapting FE-meshes to real, 3D surface detected geometry data is described hereafter (Fig. 4).

The procedure of the FE-mesh adaption to the deformed shape, which exists due to deformations in the production process, is based on the existing FE-mesh of the component. The mesh, generated with the ideal CAD-model, is saved in an ANSYS input file, which contains all nodes with its coordinates, the elements and the defined boundary conditions.

Based on the initial FEA input-file, the deviation at each surface node is measured. To determine the deviations of the nodes of the mesh, the coordinates of the created FE-mesh are used. For every coordinate of the FE-nodes, a comparison point is created on the ideal CAD-model using the software PolyWorks. With

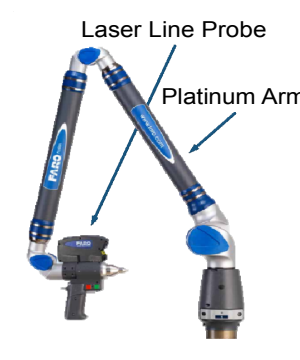

(a)

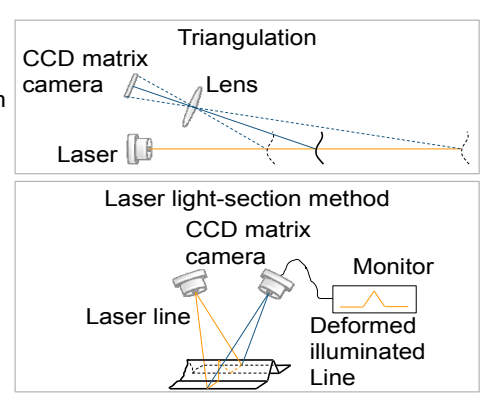

(b)
Fig. 3 (a): FARO Laser Line Probe [14] and (b): basic principle of triangulation and light-section method [15]. 


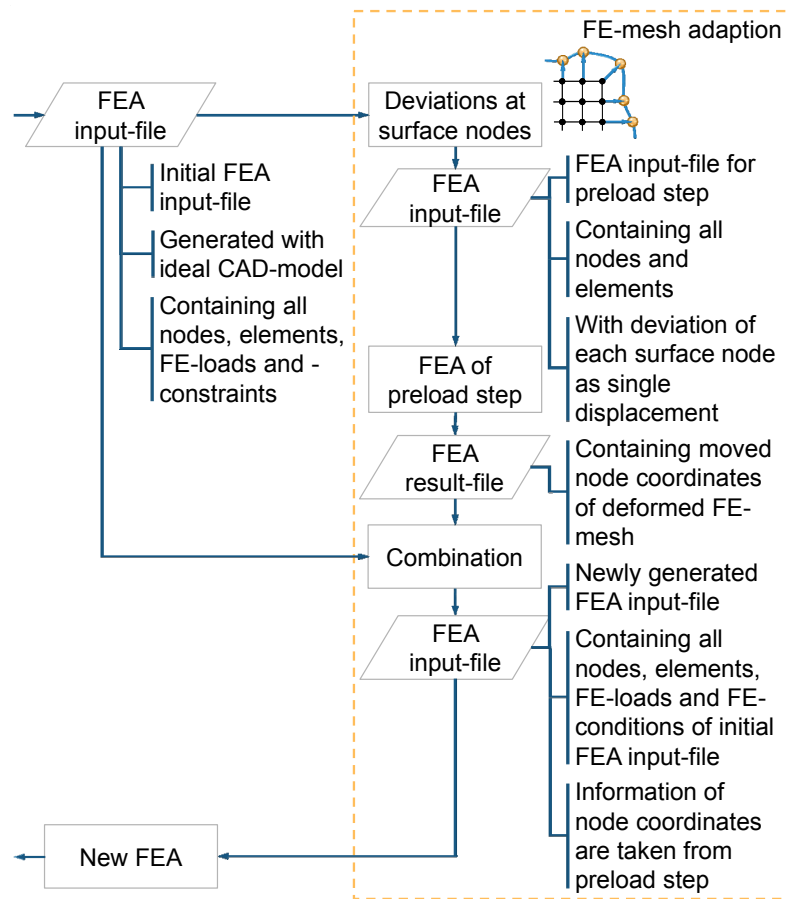

Fig. 4 Process of FE-mesh adaption.

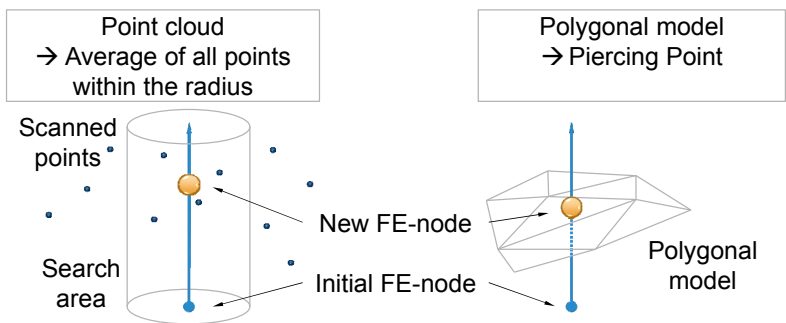

Fig. 5 Determining the deviation of one single FE-node when using point cloud scan data or a polygonal model as a real object.

such a comparison point, the deviations between the CAD-model (ideal model, basis for the FE-mesh) and the 3D surface scan (real component data) are identified by measuring the difference. For this measurement, the scanned real component can either be a point cloud or a polygonal model. To determine the deviations of these measurement points, a vector normal to its face is calculated for every node. In addition, if there is a point cloud, the search for scanned points within a predefined (and adaptable) radius of the normal vector of the node will be conducted. The average of all located points is used to move the node along the normal vector. Some scanner systems create a polygonal model during the scan process directly, so in this case, if there is a polygonal model instead of a point cloud, the piercing point of the normal vector of one polygonal surface will be used as the new position of the node (Fig. 5). The deviation of the real component to the CAD-model can be identified with this method. Thus, the existing surface nodes can be adapted in its shape to get a deviation afflicted FE-mesh with the values of the comparison points [16].

For a good fitting of the detected point cloud to the ideal CAD-model, both data sets have to be registered. This means the geometric information of the CAD-model and the scan data must be superimposed properly. If it is impossible to use reference areas (surfaces, tags), registration will be performed with a best-fit-algorithm [17]. The best-fit algorithm searches iteratively for the minimum distance between every scan point of the point cloud and the next geometry until it reaches a defined cancellation criterion (minimum value of all distances or amount of iterations).

A new input file for a preload step is created with the obtained information of the deviations of the surface nodes. This contains the initial mesh, but none of the boundary conditions. Instead, the deviation of every surface node is applied as a displacement. This preload step is necessary to avoid poor elements or even errors in the topology, when performing an analysis with volume elements to get a working mesh. This is especially the case when deviations exceed the element size (Fig. 6).

Finally, the preload step results in a mesh with the deformed, real existing shape of the prototype, respectively the manufactured component. A complete new ANSYS input-file is generated with the created mesh coordinates and the remaining information of the initial FEA input-file, like nodes (node numbering and node coordinates), elements (element numbering and containing nodes), FE-loads and FE-conditions. This data set can then be used to perform a new simulation within ANSYS containing all initially defined conditions on the ideal CAD-model, but with a mesh, adapted to real, 3D surface scanned geometry data. 


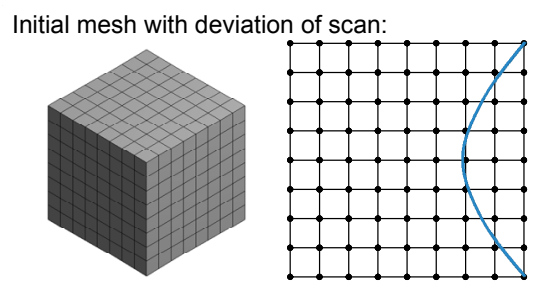

Mesh with adapted surface nodes:

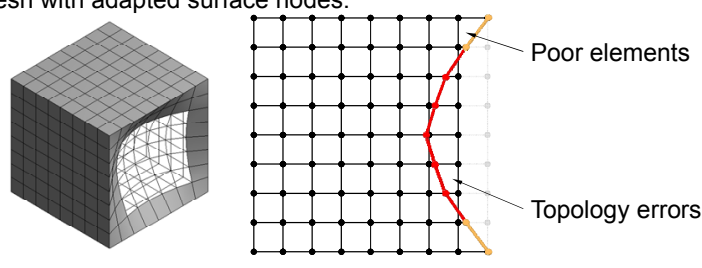

Mesh with adapted surface nodes and preload step (qualitatively):
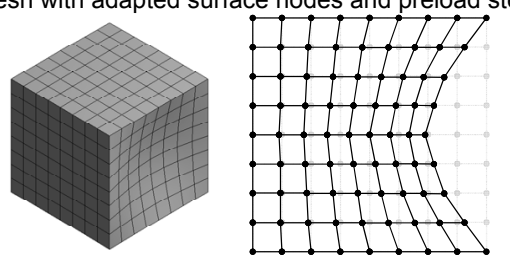

Fig. 6 Necessity of the preload step to get a working FE-mesh.

\section{Implementation and Application Using Examples}

Using a simple example of a round beam, fixed on one side and loaded with a force on the other side (Fig. 7), the functionality of the FE-mesh adaption process is to be applied and verified.

At first, a mesh of solid hexaeder elements, each containing 20 nodes, is generated from the ideal geometry of the CAD-model. This finally results in a mesh consisting of 1,470 elements with a total of 7,356 nodes.

The real component is scanned with a laser line scanner. The detected point cloud is automatically tessellated within PolyWorks. Therefore, the polygonal model is the basis for the comparison of ideal and real geometry and the FE-mesh adaption. A false-color plot of the comparison highlights deformations in the central area of the beam.

Finally, the algorithm of FE-mesh adaption is used to create a new file with a mesh adapted to the scan data of the beam (Fig. 8).

A comparison of the element quality, the aspect ratio and the maximum corner angle of the initial and the

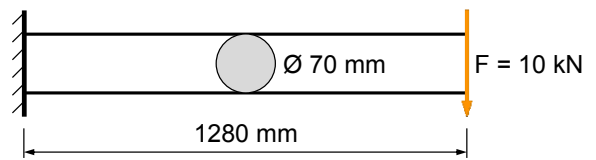

Fig. 7 Sketch of task of the example round beam.

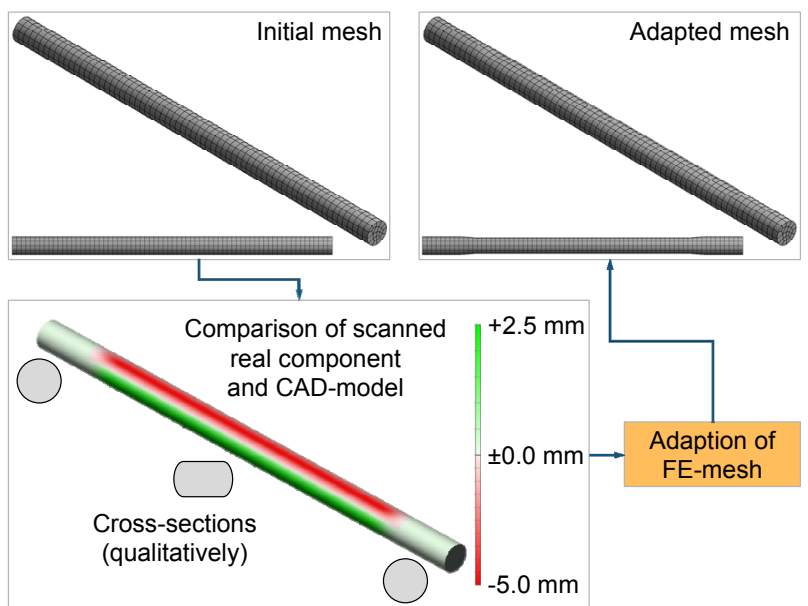

Fig. 8 Comparison of real component and CAD-model and initial and adapted meshes.

Table 1 Comparison of mesh metric between initial and adapted mesh.

\begin{tabular}{|l|r|l|l|}
\hline Mesh metric & Min & 0.7194031128 & 0.7165165822 \\
\hline \multirow{3}{*}{ Element quality } & Max & 0.9964004911 & 0.9997368602 \\
\cline { 2 - 4 } & Average & 0.8751553018 & 0.8652295529 \\
\hline \multirow{3}{*}{ Aspect ratio } & Min & 1.1955 & 1.1946 \\
\cline { 2 - 4 } & Max & 2.0749 & 2.2577 \\
\cline { 2 - 4 } & Average & 1.7614142857 & 1.7974571429 \\
\hline \multirow{3}{*}{ Maximum corner angle } & Min & $102.74^{\circ}$ & $100.23^{\circ}$ \\
\cline { 2 - 4 } & Max & $125.77^{\circ}$ & $133.55^{\circ}$ \\
\cline { 2 - 4 } & Average & $116.04^{\circ}$ & $117.06^{\circ}$ \\
\hline
\end{tabular}

adapted mesh is necessary and useful for checking the mesh metric after the process (Table 1). All in all, no significant deterioration of the mesh can be identified.

A comparison of the results then again reveals differences between both simulations (Fig. 9). As expected, the calculation of the total deformation shows a higher displacement with the adapted mesh. Although the maximum equivalent stress (von-Mises) of the adapted geometry is equal to the initial mesh, but the area with a high value has increased markedly.

In another example, the effects of a too small hold down force on a deep-drawn cupping are examined. Here the flange of the cupping with internal pressure shall be pushed down to a plain basis to obtain tightness 


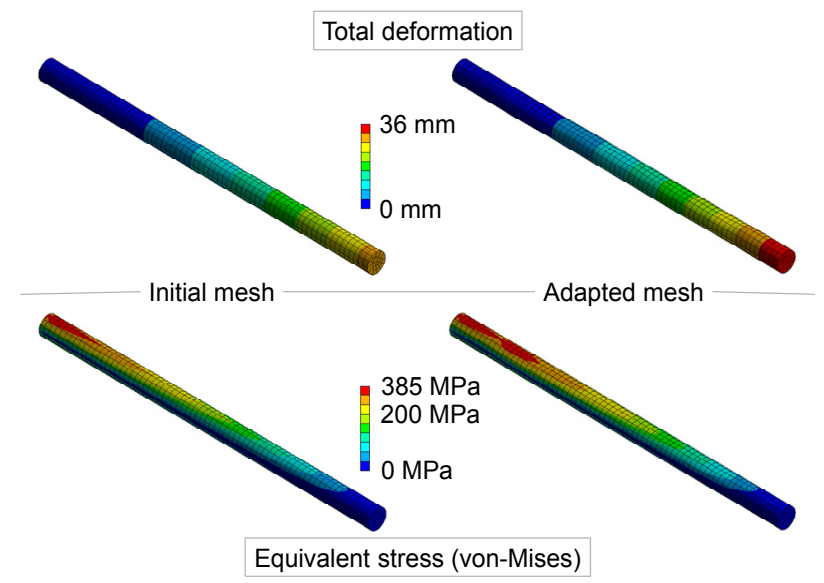

Fig. 9 Comparison of results between ideal and real geometry.

Fig. 10 Sketch of task of the example deep-drawn cupping.

between basis and flange (Fig. 10). The question to clarify is the increase of force, which is used to obtain tightness.

In this case, the mesh of the cupping consists of 9,176 shell elements, each containing eight nodes, that means 15,446 nodes in total.

The further procedure of preprocessing the FE-mesh adaption is almost similar to the first example. Comparing CAD-model and surface scan, the wavelike deformation in the flange area can be seen (Fig. 11).

A new analysis is performed using the adapted mesh (Fig. 12). The reaction force at the basis is calculated within this simulation with a value of about $48.7 \mathrm{kN}$. This additional force has to be applied to the actual hold down force of the ideal geometry model, when the flange of the cupping is deformed like this due to the production process.

\section{Future Fields of Research}

To make this application useable in general, the transfer and analysis of the solver input file into a neutral format is pursued. When choosing a format, the three points of neutral CAx-formats according to [18]

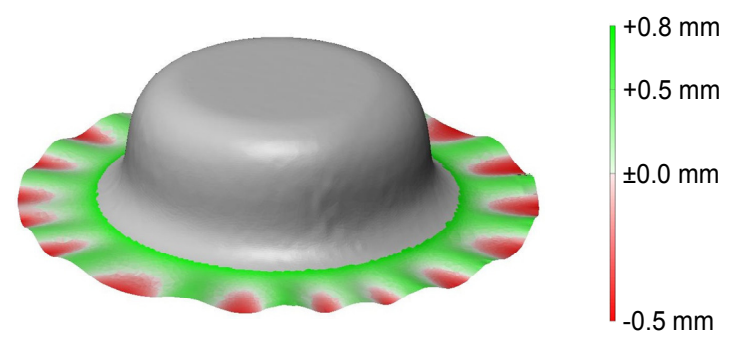

Fig. 11 Deviations in the flange area compared to a plain.

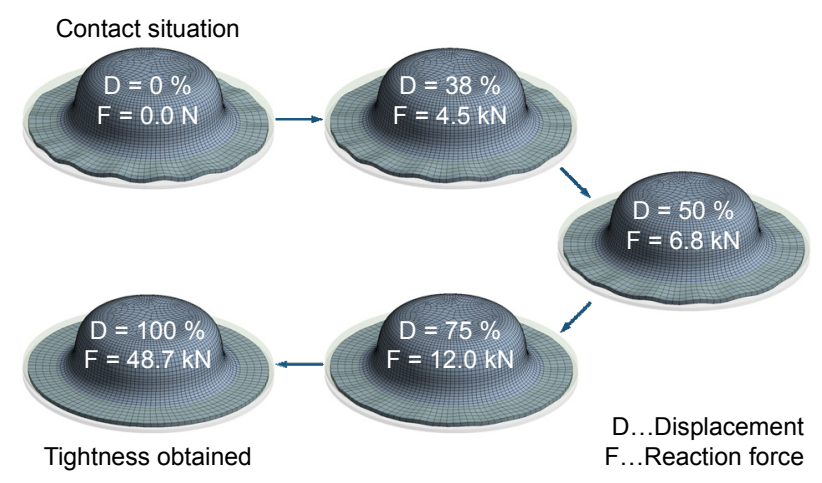

Fig. 12 Steps of simulation with adapted mesh.

have to be maintained: data consistency, size of data and flexibility. The standard XML-format (Extensible Markup Language) is suitable for this purpose as it is generally understandable and data can easily be exchanged in this format [19].

To support the decision making process of design engineers, the question whether the existing deviations are relevant for the simulation or not, a knowledge base shall be developed and included in the process as illustrated in Fig. 2. This knowledge base contains the tolerances of the production process and permissible deviations as well as comparative analysis of similar and/or previous versions of the component.

The process itself should offer the user the opportunity to select and adapt only the required nodes of special interest, instead of all nodes. This limitation on specific areas of special interest leads to a reduction in computational hardware requirements and time when the model is huge and contains thousands of nodes.

In addition, it is a present concern to substitute the tree-dimensional surface scan of the real component with a simulated model (for example using software for 
manufacturing simulation like AutoForm or Moldflow). Consequently, the engineer has the chance to analyze the component with real geometry data in an early phase of the product development process, even before there is a deformed component as a prototype at hand. Within the context of a digital mock-up [20], the renunciation of the real manufactured prototype has to be aspired.

Finally, the aim to automate the approach of the FE-mesh adaption completely should be achieved in order to minimize the time for preparing the simulation and to ensure a low susceptibility to errors.

\section{Conclusions}

To sum up, every produced component differs in its geometry compared to the ideal model of the CAD-system. As shown in the prior examples, these deviations due to the production process can trigger various influences on the results of performed analysis. When using the reverse engineering process to represent those deviations, the amount of time used for surface reconstruction and a whole FEA preprocessing would be disadvantageous. Instead, the practical approach of adapting FE-meshes to real, 3D surface detected geometry data described in this paper gives the engineer an easy to use opportunity to get closer to realism with the FE-simulation models. Once the real geometry in form of a prototype is available, a new simulation can be easily performed without losing a lot of time for further preprocessing.

\section{Acknowledgement}

The presented approach was researched as part of FORPRO $^{2}$, the Bavarian research alliance for efficient product and process development due to knowledge based simulation [21]. The aim of the FORPRO $^{2}$-project is to increase the efficiency of virtual product and manufacturing process development through the creation of a framework with linked simulation environments and expertise to design optimization and risk assessment of products.
Special thanks are directed to the BFS (Bayerische Forschungsstiftung) for financial support of the whole research network [22].

\section{References}

[1] Bartschlager, J., Dillinger, J., Escherich, W., Guenter, W., Ignatowitz, E., Oesterle, St., Reisser, L., Stephan, A., Vetter, R., and Wienecke, F. 2013. Expertise Metal. Haan-Gruiten: Europa Lehrmittel. (In German)

[2] Birkert, A., Haage, St., and Straub, M. 2013. Metal Forming Production of Complex Body Parts-Technical Design of Drawing Plants. Berlin: Springer. (In German)

[3] Nee, A. Y. C. 2014. Handbook of Manufacturing Engineering and Technology. London: Springer.

[4] Thoma, Ch., Volk, W., Branner, G., and Eibisch, H. 2013. Simulation Based Optimization of the Dimensional Accuracy for Thin-Walled Structural Components in Aluminium High Pressure Die Casting. Wien: Casting Review. (In German)

[5] Gebhardt, Ch. 2011. Applied FEA Using ANSYS Workbench-An Introduction to Linear and Non-linear Mechanics. Munich: Hanser. (In German)

[6] Rosemann, B., and Freiberger, St. 2012. "Reverse Engineering." In Handbook of Engineering Design, edited by Rieg, F., and Steinhilper, R. Munich: Hanser. (In German)

[7] Raja, V., and Fernandes, K. J. 2008. "Reverse Engineering-An Industrial Perspective." London: Springer.

[8] Schoene, Ch. 2009. Reverse Engineering for Free-Form Faces. Muenchen: Dr. Hut. (In German)

[9] Katona, S., Koch, M., and Wartzack, S. 2015. "Generating Hybrid Geometry Models for More Precise Simulations by Combining Parametric CAD-Models with 3D Surface Detected Geometry Data." Presented at the 20th International Conference on Engineering Design (ICED15), Milan, Italy.

[10] Piegl, L., and Tiller, W. 1997. The NURBS-Book. Berlin: Springer.

[11] Fagan, M. J. 1992. Finite Element Analysis-Theory and Practice. New York: Longman Scientific \& Technical.

[12] Faro Europe GmbH \& Co. KG 2010. “Technical Data Sheet." Accessed January 12, 2014. http://www2.faro.com/site/resources/share/555.

[13] Toenshoff, H. K., and Inasaki, I. 2001. "Sensors Application Volume 1-Sensors in Manufacturing." Weinheim: Wiley-VCH.

[14] Faro Europe GmbH \& Co. KG 2010. "Products-Faro Platinum Arm with Laser Line Probe." Accessed August 4, 2014. https://faro.blob.core.windows.net/sitefinity/ tech-sheets/04ref201-275-de---faro-laser-scanarm-v3-tec 


\section{Structural Mechanics Analysis Using an FE-Mesh Adaption to Real, 3D Surface Detected Geometry Data}

h-sheet.pdf? sfvrsn=6.

[15] Katona, S., Koch, M., and Wartzack, S. 2014. "Reverse Enginerring-Process, Technologies and Applications." Presented at the16th Bayreuther Day of 3D Engineering Design, University Bayreuth. (In German)

[16] Katona, S., Kestel, P., Koch, M., and Wartzack, S. 2014. "From Ideal- to Real-Model-Simulating Components with Manufacturing Deviations Using Automated FE-Mesh Adaption.” In Design Develop Experiencee-Contributions to Virtual Product Development and Engineering Design, edited by Ralph, S. Dresden: TUD press. (In German)

[17] Chen, L. M. 2012. Digital Functions and Data Reconstruction-Digital Discrete Methods. New York: Springer.

[18] Troll, A. 2014. "3D Data Exchange and Data Formats in the Product Development." In Handbook of Engineering Design, edited by Rieg, F., and Steinhilper, R. Munich:
Hanser. (In German)

[19] MacIntyre, P., Canchilla, B., and Gogala, M. 2011. Pro PHP Programming. New York: Apress.

[20] Scholz, E., Burckhardt, Ch., and Dietrich, S. 2006. "Digital Mock-Ups in Product Development." Accessed January 12 ,

2015. https://fbme.htwk-leipzig.de/fileadmin/fbme/ informationen/TVorstellung/DMU.pdf. (In German)

[21] FORPRO ${ }^{2}$. 2013. "Research Alliance FORPRO ${ }^{2}$." Accessed November 26, 2014. http://www.forpro2.tum.de/index.php?id=5\&L=1.

[22] BFS. 2013. "Bavarian Research Alliance for Efficient Product and Process Development Due to Knowledge Based Simulation." Accessed November 26, 2014. http://www.forschungsstiftung.de/Projekte/Details/Forsch ungsverbund-fuer-effiziente-Produkt-und-Prozessentwick lung-durch-wissensbasierte-Simulation-FORPRO.html. (In German) 